Pacific Journal of Mathematics

A PROBLEM OF PANZER 


\section{ON A PROBLEM OF DANZER}

\section{R. P. BAmbah AND A. C. Woods}

By a Danzer set $S$ we shall mean a subset of the $n$-dimensional Euclidean space $R_{n}$ which has the property that every closed convex body of volume one in $R_{n}$ contains a point of $S$. L. Danzer has asked if for $n \geqq 2$ there exist such sets $S$ with a finite density. The answer to this question is still unknown. In this note our object is to prove two theorems about Danzer sets.

If $\Lambda$ is a $n$-dimensional lattice, any translate $\Gamma=\Lambda+p$ of $\Lambda$ will be called a grid $\Gamma$; $\Lambda$ will be called the lattice of $\Gamma$ and the determinant $d(\Lambda)$ of $\Lambda$ will be called the determinant of $\Gamma$ and will be denoted by $d(\Gamma)$. In $\S 2$ we prove

Theorem 1. For $n \geqq 2$, a Danzer set cannot be the union of a finite number of grids.

Let $S$ be a Danzer set and $X>0$ a positive real number. Let $N(S, X)$ be the number of points of $S$ in the box $\max _{1 \leqq i \leqq n}\left|x_{i}\right| \leqq X$. Let $D(S, X)=N(S, X) /(2 X)^{n}$. In $\S 3$ we prove

Theorem 2. There exist Danzer sets $S$ with $D(S, X)=$ $0\left((\log X)^{n-1}\right)$ as $X \rightarrow \infty$.

The case $n=2$ of the theorem is known, although no proof seems to have been published. The referee has pointed out that a lower bound of 2 can easily be established for the density of a Danzer set in $n=2$, but the authors are unaware of any further results in this direction.

2. Proof of Theorem 1. We shall assume throughout that $n \geqq 2$. It is obvious that if $S$ is a Danzer set and $T$ is a volume preserving affine transformation of $R_{n}$ onto itself, then $T^{\prime}(S)$ is also a Danzer set.

Let $S_{1}, S_{2}, \cdots$ be a sequence of sets in $R_{n}$. Let $S$ be the set of points $X$ such that there exists a subsequence $S_{i_{1}}, S_{i_{2}}, \cdots$ of $\left\{S_{r}\right\}$ and points $X_{i_{r}} \in S_{i_{r}}$, such that $X_{i_{r}} \rightarrow X$ as $r \rightarrow \infty$. We write

$$
S=\lim _{r \rightarrow \infty} S_{r}=\lim S_{r} .
$$

Lemma 1. Let $\left\{S_{r}\right\}$ be a sequence of Danzer sets in $R_{n}$. Then $S=\lim S_{r}$ is also a Danzer set.

Proof. Let $K$ be a closed convex body of Volume 1. Then for 
each $r, K \cap S_{r} \neq \phi$, so that for each $r$, there exists $X_{r} \in K \cap S_{r}$. Since $K$ is compact, $\left\{X_{r}\right\}$ has a convergent subsequence $\left\{X_{i_{r}}\right\}$ converging to a point $X$ in $K \cap S$.

Lemma 2. Let $S^{(j)}=\lim _{r \rightarrow \infty} S_{r}^{(j)}, j=1, \cdots, k$. Then

$$
\bigcup_{j=1}^{k} S^{(j)}=\lim _{r \rightarrow \infty}\left(\bigcup_{j=1}^{k} S_{r}^{(j)}\right) \text {. }
$$

Proof. $X \in \cup S^{(j)} \Rightarrow X \in S^{(j)}$ for some $j$, say $j=j_{0} \Rightarrow$ there exist a subsequence $\left\{S_{i_{r}}^{\left(j_{0}\right)}\right\}$ of $\left\{S_{i_{r}}^{\left(j_{0}\right)}\right\}$ and points $X_{i_{r}} \in S_{i_{r}}^{\left(j_{0}\right)}$ such that $X_{i_{r}} \rightarrow$ $X \Rightarrow X_{i_{r}} \in \cup S_{i_{r}}$ and $X_{i_{r}} \rightarrow X \Rightarrow X \in \lim _{r \rightarrow \infty}\left(\bigcup_{j=1}^{k} S_{r}^{(j)}\right)$. Thus $\cup S^{(j)} \subset$ $\lim \left(\bigcup_{j=1}^{k} S_{r}^{(j)}\right)$. Let $X \in \lim \left(U_{j=1}^{k} S_{r}^{(i)}\right)$. Then there exists a sequence $\left\{i_{r}\right\}$ of natural numbers and $X_{i_{r}} \in \bigcup_{j=1}^{k} S_{i_{r}}^{(j)}$ such that $X_{i_{r}} \rightarrow X$. Since $k$ is finite, there exists a $j=j_{0}$ say, and an infinite subsequence $k_{r}$ of $i_{r}$ such that $X_{k_{r}} \in S_{k_{r}}^{\left(j_{0}\right)}$. Then $X_{k_{r}} \rightarrow X$ and $X \in S^{\left(j_{0}\right)}$, so that $X \in \cup S^{\left(j_{0}\right)}$ and $\lim _{r \rightarrow \infty}\left(\bigcup_{j=1}^{k} S_{s}^{(j)}\right) \subset \cup S^{(j)}$.

This completes the proof of the lemma.

Lemma 3. Let $\Gamma_{1}, \Gamma_{2}, \cdots$ be a sequence of grids in $R_{n}$ with equal determinants $d\left(\Gamma_{r}\right)=\Delta$. Then $\left\{\Gamma_{r}\right\}$ has a subsequence $\left\{\Gamma_{i_{r}}\right\}$, such that $\lim _{r \rightarrow \infty} \Gamma_{i_{r}}$ is either a grid or is contained in a hyperplane.

Proof. If $\lim _{r \rightarrow \infty} \Gamma_{r}=\phi$, there is nothing to prove. Assume, therefore, that $\Gamma=\lim _{r \rightarrow \infty} \Gamma_{r} \neq \phi$. Let $X \in \Gamma$. Then there exists a subsequence $\left\{i_{r}\right\}$ of natural numbers and points $X_{i_{r}} \in \Gamma_{i_{r}}$, such that $X_{i_{r}} \rightarrow X$. Then $\Lambda_{i_{r}}=\Gamma_{i_{r}}-X_{i_{r}}$ is a sequence of homogeneous lattices and $\lim \Gamma_{i_{r}}=X+\lim \Lambda_{i_{r}}$. Therefore, it is enough to prove the theorem for lattices.

Let $\left\{\Lambda_{r}\right\}$ be a sequence of lattices with determinants $d\left(\Lambda_{r}\right)=\Delta$, independent of $r$. Let $\mu_{1}\left(\Lambda_{r}\right), \cdots, \mu_{n}\left(\Lambda_{r}\right)$ be the successive minima of the Euclidean distance with respect to $\Lambda_{r}$, i.e., $\mu_{i}\left(\Lambda_{r}\right)=\inf \mu:$ such that $|X|<\mu$ has $i$ linearly independent points of $\Lambda_{r}$.

Suppose, first, that there exists $\delta>0$, such that $\mu_{1}\left(\Lambda_{r}\right) \geqq \delta$ for infinitely many $r$. Then a subsequence satisfies the conditions of Mahler's compactness theorem and has a subsequence convergent in the sense of Mahler (see, e.g., Cassels [2]). The last subsequence converges to the limiting lattice in our sense also.

We may, therefore, assume $\mu_{1}\left(\Lambda_{r}\right) \rightarrow 0$ as $r \rightarrow \infty$. Since

$$
\mu_{1}\left(\Lambda_{r}\right) \cdots \mu_{n}\left(\Lambda_{r}\right) \geqq \frac{2^{n}}{n !} \cdot \frac{1}{J_{n}},
$$

where $J_{n}$ is the volume of the sphere $|X|<1$, (see, e.g., Cassels [2]), 
and since $n \geqq 2$, it follows that $\mu_{n}\left(\Lambda_{r}\right) \rightarrow \infty$ as $r \rightarrow \infty$. For each $r$, let $P_{r_{1}}, \cdots, P_{r_{n}}$ be points such that $\left|P_{r_{i}}\right|=\mu_{i}\left(\Lambda_{r}\right)$. Let $\pi_{r}$ be the plane through $0, p_{r_{1}}, \cdots, p_{r_{n-1}}$. It is easily seen that there exists a subsequence $\left\{\Lambda_{i_{r}}\right\}$ of $\left\{\Lambda_{r}\right\}$ such that the sequence $\left\{\pi_{i_{r}}\right\}$ converges to a plane $\pi$. We assert that $\lim _{r \rightarrow \infty}\left\{\Lambda_{i_{r}}\right\} \subset \pi$. For, let $X \in \lim _{r \rightarrow \infty} \Lambda_{i_{r}}$. Then $X=\lim X_{k_{r}}$, where $k_{r}$ is a subsequence of $i_{r}$ and $X_{k_{r}} \in \Lambda_{k_{r}}$. There exists $M$ independent of $k_{r}$, such that $\left|X_{k_{r}}\right| \leqq M$ for all $k_{r}$. Also

$$
X_{k_{r}}=g_{r, 1} P_{k_{r}, 1}+\cdots+g_{r, n} P_{k_{r}, n}, g_{r, i} \text { real , }
$$

and if $g_{r, n} \neq 0$ then $\left|X_{k_{r}}\right| \geqq \mu_{n}\left(\Lambda_{k_{r}}\right)$. Since $\mu_{n}\left(\Lambda_{k_{r}}\right) \rightarrow \infty$ as $r \rightarrow \infty$, $g_{r, n}=0$ for all large $r$ and $X \in \pi$. This proves the lemma

Lemma 4. Let $\left\{\pi_{i}\right\}$ be a sequence of hyperplanes. Then $\left\{\pi_{i}\right\}$ has a subsequence $\left\{\pi_{i_{\mu}}\right\}$ whose limit lies in a hyperplane.

Proof. If $\pi=\lim _{i \rightarrow \infty} \pi_{i}=\dot{\phi}$ then there is nothing to prove. Assume, therefore, $X \in \pi$. Then there is a subsequence $\left\{k_{r}\right\}$ of natural numbers and points $X_{k_{r}} \in \pi_{k_{r}}$ such that $X_{k_{r}} \rightarrow X$. The planes $\hat{\pi}_{k_{r}}=$ $\pi_{k_{r}}-X_{k_{r}}$ pass through 0 and have a subsequence $\hat{\pi}_{i_{r}}$ which converges to a plane $\hat{\pi}$ say. Then $\lim _{r \rightarrow \infty} \pi_{i_{r}}=\hat{\pi}+X$. This proves the lemma.

Proof of Theorem 1. We shall prove more, namely, a Danzer set cannot be the union of a finite number of hyperplanes and a finite number of grids.

Let $S=\bigcup_{i=1}^{r} \pi_{i} \bigcup_{j=1}^{t} \Gamma_{j}$ be a Danzer set, such that $\pi_{i}$ are hyperplanes and $\Gamma_{j}$ are grids. Let $t \geqq 1$. Let $X \neq Y, X, Y \in \Gamma_{1}$. For each positive integer $k$, let $T_{k}$ be a volume preserving affine transformation such that $T_{k}(X)=X$ and $\left|T_{k}(Y)-X\right|=k^{-1}|Y-X|$. Since $n \geqq 2$, such transformations exist. For each $k, T_{k}(S)$ is a Danzer set, and by Lemma 1 , so is the limit of every subsequence of $\left\{T_{k}(S)\right\}$. By Lemmas 3 and 4 we can choose a subsequence $\left\{T_{k_{r}}\right\}$ of $\left\{T_{k}\right\}$ such that each $\lim _{t \rightarrow \infty} T_{k_{r}}\left(\pi_{i}\right)$ lies in a hyperplane, while each $\lim _{t \rightarrow \infty} T_{k_{r}}\left(\Gamma_{j}\right)$ is either a grid or lies in a hyperplane. Since

$$
\lim _{r \rightarrow \infty} T_{k_{r}}(S)=\bigcup_{i=1}^{t} \lim T_{k_{r}}\left(\pi_{i}\right) \bigcup_{j=1}^{t} \lim T_{k_{r}}\left(\Gamma_{j}\right)
$$

and $\lim T_{k_{r}}\left(\Gamma_{1}\right)$ is in a hyperplane, the Danzer set $\lim T_{k_{r}}(S)$ lies in the union of a finite number of hyperplanes and $t_{1}<t$ grids, so that we have (by increasing $T_{k_{r}}(S)$ if necessary) a Danzer set consisting of a finite number of hyperplanes and $t_{1}<t$ grids. Repeating this process a number of times we obtain a Danzer set that is the union of a finite number of hyperplanes. This can easily be seen to lead to a contradiction which proves the theorem. 
3. Proof of Theorem 2. Let $K$ be a closed convex body in $R_{n}$. The set $S \subset R_{n}$ is said to be a covering set for $K$ if $R_{n} \subset \bigcup_{A \in S}(K+A)$. The set $S$ contains a point of each translate of $K$ if and only if $S$ is a covering set for $-K$. Clearly a set $S$ is a Danzer set if and only if it is a covering set for each closed convex body of volume one. Therefore, in order to prove a given set $S$ is a Danzer set, it is enough to prove that for every closed convex body $K$ of volume one, $S$ contains a covering set for $K$.

If $\Gamma$ is a grid with lattice $\Lambda$, then it is easy to see that $\Gamma$ is a covering set for $K$ if and only if $\Lambda$ is.

Let $\pi$ be a parallelepiped. Let $A_{0}$ be one of its vertices and $A_{1}, \cdots, A_{n}$ be the $n$ vertices joined to $A_{0}$ by edges of $\pi$. Let $\Lambda$ be the lattice generated by $A_{1}-A_{0}, \cdots, A_{n}-A_{0}$. By the grid generated by $\pi$ we shall mean the grid $\Lambda+A_{0}$. It is easily seen that if a closed convex body $K$ contains a parallelepiped which generates a grid $\Gamma$, then $\Gamma$ is a covering set for $K$.

A lattice $\Lambda$ will be called rectangular if it consists of points $\left(\alpha_{1} u_{1}, \cdots, \alpha_{n} u_{n}\right)$, where $\alpha_{i}$ are fixed positive real numbers and $u_{i}$ take integral values. A grid $\Gamma$ will be called rectangular if its lattice is rectangular.

Let $\alpha_{1}, \cdots, \alpha_{n}$ be positive real numbers. Let $\Gamma_{\alpha}$ be the grid generated by the parallelepiped $\left|x_{i}\right| \leqq \alpha_{i}$. Let $B$ be a box $\left|x_{i}\right| \leqq \beta_{i}$, where $\beta_{i} \geqq \alpha_{i}$ for $i=1, \cdots, n$. Then $\Gamma_{\alpha}$ is clearly a covering set for $B$.

Let $K$ be a closed convex body of volume one. Let $K_{1}$ be the steiner symmetrical of $K$ with respect to the plane $x_{1}=0$. Let $K_{2}$ be the steiner symmetrical of $K_{1}$ with respect to $x_{2}=0$ and so on. Then $K_{n}$ is symmetrical about all the coordinate planes and has volume one. We next have

LEMMA 5. If a rectangular lattice $A$ is a covering set for $K_{n}$, then it is a covering set for $K$ also.

(The lemma and its proof are easy adaptions of Lemma 2 of Sawyer (3). For completeness, we give the proof below).

Proof. Let $\Lambda$ be the rectangular lattice consisting of points $\left(\alpha_{1} u_{1}, \cdots, \alpha_{n} u_{n}\right), \alpha_{i}>0$ fixed real numbers and $u_{i}$ running over the set of integers. It is enough to prove that if $\Lambda$ is a covering set for $K_{1}$, then it is a covering set for $K$ also.

Let $\Lambda_{1}=$ subset of $\Lambda$ in the plane $x_{1}=0$. The sets $K_{1}+\Lambda$ cover $R_{n}$. We assert each line $x_{2}=a_{2}, \cdots, x_{n}=a_{n}$ meets $K_{1}+P$ is a segment of length at least $\alpha_{1}$ for some $P \in \Lambda_{1}$. Such a line meets only a finite number of translates $K_{1}+P_{s}, P_{s} \in \Lambda_{1}$, each of them in a seg- 
ment $\left|x_{1}\right| \leqq b_{s}$ and hence meets $K_{1}+\Lambda_{1}$ in the segment $\left|x_{1}\right| \leqq b=$ $\max b_{s}$. If $b<\frac{1}{2} \alpha_{1}$, then $K_{1}+\Lambda$ meets the line in segments $\mid x_{1}$ $m \alpha_{1} \mid \leqq b<\frac{1}{2} \alpha_{1}$, where $m$ takes integral values. This leaves part of the line uncovered by sets $K_{1}+\Lambda$, contrary to the fact that $\Lambda$ is a covering set for $K_{1}$. Thus $b \geqq \frac{1}{2} \alpha_{1}$, i.e., $b_{s} \geqq \frac{1}{2} \alpha_{1}$ for some $s$. Therefore, the line meets $K_{1}+P_{s}$ and hence $K+P_{s}$ in a segment of length at least $\alpha_{1}$, and is therefore, covered by the sets $K+\Lambda$. Since this is true for all such lines, $\Lambda$ is a covering set for $K$.

COROLlARY. A rectangular grid $\Gamma$ which is a covering set for $K_{n}$ is also a covering set for $K$.

Because of the corollary, in oder to prove that a given set $S$ is a Danzer set, it is enough to prove that for every given closed convex body $K$ of volume one, which is symmetrical about all the coordinate planes, $S$ contains a rectangular grid $\Gamma$ which is a covering set for $K$.

Let $K$ be a closed convex body of volume one, which is symmetrical about the coordinate planes. Then $K$ contains a point $\left(a_{1}, \cdots, a_{n}\right)$, $a_{i}>0$, such that $2^{n} a_{1} \cdots a_{n} \geqq n ! / n^{n}$. (See, e.g., Sawyer [3]). Then $K$ contains a box $B_{\beta}:\left|x_{i}\right| \leqq \beta_{i}, \beta_{i} \leqq a_{i}$ with volume $2^{n} \beta_{1} \cdots \beta_{n}=n ! / n^{n}$. A covering rectangular grid of $B_{\beta}$ is automatically a covering set for $K$. Therefore, $S$ is a Danzer set if for all closed boxes $B_{\beta}$ of volume $n ! / n^{n}, S$ contains a rectangular grid $\Gamma_{\alpha}$ generated by $\left|x_{i}\right| \leqq \alpha_{i}$ with $\alpha_{i} \leqq \beta_{i}$.

We now construct a set $A$ of points $\alpha=\left(\alpha_{1}, \cdots, \alpha_{n}\right), \alpha_{i}>0$, such that for each set $\left(\beta_{1}, \cdots, \beta_{n}\right), \beta_{i}>0, \beta_{1} \cdots \beta_{n}=n ! /(2 n)^{n}=k$, say, there exists an $\alpha \in A$, such that $\alpha_{i} \leqq \beta_{i}$. Then the grid $\Gamma_{\alpha}$ will provide a convering by $B_{\beta}$ and the set $S=\bigcup_{\alpha \in A} \Gamma_{\alpha}$ will be a Danzer set.

Let $H$ be the set of point $x$ such that $x_{1} \cdots x_{n}=k, x_{i}>0$. Divide the part $x_{1}>0, \cdots, x_{n-1}>0$ of the plane $x_{n}=0$ into $n-1$ dimensional parallelepipeds $\pi_{k_{1}, \ldots, k_{n-1}}$ defined by

$$
e^{k_{i}} \leqq x_{i} \leqq e^{k_{i}+1}, i=1, \cdots, n-1,\left(k_{1}, \cdots, k_{n-1}\right) \in Z^{n-1},
$$

when $Z$ is the set of rational integers. Let $H_{k_{1}, \cdots, k_{n-1}}=\{x: x \in H$ and $\left.\left(x_{1}, \cdots, x_{n-1}\right) \in \pi_{k_{1}, \cdots, k_{n-1}}\right\}$. Then $H=\bigcup_{\left(k_{1}, \cdots, k_{n-1}\right) \in S^{n-1}} H_{k_{1}, \cdots, k_{n-1}}$. If $X \in$ $H_{k_{1}, \cdots, k_{n-1}}$, then $x_{i} \geqq e^{k_{i}}, i=1, \cdots, n-1$ and

$$
x_{n}=\frac{k}{x_{1} \cdots x_{n-1}} \geqq \frac{k}{e^{k_{1}+\cdots+k_{n-1}+n-1}} .
$$

Let

$$
\alpha=\alpha_{k_{1}, \cdots, k_{n-1}}=\left(e^{k_{1}}, \cdots, e^{k_{n-1}}, \frac{k}{e^{k_{1}+\cdots+k_{n-1}+n-1}}\right)
$$

Then $\Gamma_{\alpha}$ is a grid of determinant $2^{n} k / e^{n-1}$. Let 


$$
A=\left\{\alpha_{k_{1}, \cdots, k_{n-1}}:\left(k_{1}, \cdots, k_{n-1}\right) \in Z^{n-1}\right\} .
$$

For each $\beta=\left(\beta_{1}, \cdots, \beta_{n}\right) \in H_{k_{1}, \cdots, k_{n-1}}, \alpha_{k_{1}, \cdots, k_{n-1}} \in A$ has the property that $\Gamma_{\alpha}$ is a covering set for $B_{\beta}$. Therefore $S=\mathrm{U}_{\alpha \in A} \Gamma_{\alpha}$ is a Danzer set. To prove Theorem 2, it will be enough to prove $D(S, X)=$ $O\left((\log X)^{n-1}\right)$, as $X \rightarrow \infty$.

Let $B(X)$ be the box $\left|x_{i}\right| \leqq X$. Since $N(S, X), N\left(\Gamma_{\alpha}, X\right)$ denote the number of points of $S$ and $\Gamma_{\alpha}$, respectively, in $B(X)$, it follows that

$$
\text { (*) } \quad N(S, X) \leqq \sum_{\alpha \in A} N\left(\Gamma_{\alpha}, X\right) .
$$

If $\alpha=\alpha_{k_{1}, \cdots, k_{n-1}} \in A$, then the points of $\Gamma_{\alpha}$ have coordinates

$$
\begin{aligned}
& \left(e^{k_{1}} u_{1}, e^{k_{2}} u_{2}, \cdots, e^{k_{n-1}} u_{n-1}, \frac{k}{e^{k_{1}+\cdots+k_{n-1}+n-1}} u_{n}\right) \\
= & \left(e^{k_{1}} u_{1}, e^{k_{2}} u_{2}, \cdots, e^{k_{n-1}} u_{n-1}, k e^{l} u_{n}\right),
\end{aligned}
$$

say, where $u_{i}$ are odd integers. If $\Gamma_{\alpha} \cap B(X) \neq \phi$, then

$$
e^{k_{1}} \leqq X, \cdots, e^{k_{n-1}} \leqq X, k e^{l} \leqq X,
$$

so that for

$$
\begin{aligned}
i=1,2, \cdots, n-1, e^{k_{i}} & \geqq \frac{k}{e^{n-1}} \cdot \frac{e^{k_{i}}}{e^{k_{1}+\cdots+k_{n-1}}} \cdot \frac{1}{X} \\
& \geqq \frac{k}{e^{n-1}} \cdot \frac{1}{X^{n-1}} \cdot
\end{aligned}
$$

Therefore,

$$
\begin{aligned}
\Gamma_{\alpha} \cap B(X) \neq \dot{\phi} & \Rightarrow \frac{k}{(e X)^{n-1}} \leqq e^{k_{i}} \leqq X, \text { for } i=1, \cdots, n-1 \\
& \Rightarrow \log k-(n-1)(1+\log X) \leqq k_{i} \leqq \log X \\
& \text { for } i=1, \cdots, n-1 .
\end{aligned}
$$

Therefore, the number $\nu(X)$ of $\alpha$ for which $\Gamma_{\alpha} \cap B(X) \neq \phi$, satisfies

$$
\begin{aligned}
\nu(X) & \leqq(n(1+\log X)-\log k)^{n-1} \\
& =O(\log X)^{n-1} .
\end{aligned}
$$

If $\Gamma_{\alpha} \cap B(X) \neq \phi$, then the number $N\left(\Gamma_{\alpha}, X\right)$ of points of $\Gamma_{\alpha}$ in $B(X)$ is the number of points $\left(u_{1}, \cdots, u_{n}\right) \in Z^{n}, u_{i}$ odd, with

$$
-X \leqq u_{i} e^{k_{i}} \leqq X, i=1, \cdots, n-1
$$

and

$$
-X \leqq u_{n} \frac{k}{e^{k_{1}+\cdots+k_{n-1}+n-1}} \leqq X
$$


Writing $[\xi]$ for the largest integer $\leqq \xi$, we have

$$
\begin{aligned}
N\left(\Gamma_{\alpha}, X\right) & =\left(\prod_{\imath=1}^{n-1} 2\left[\frac{1}{2}\left(\frac{X}{e^{k_{i}}}+1\right)\right]\right) 2\left[\frac{1}{2}\left(\frac{X e^{k_{1}+\cdots+k_{n-1}+n-1}}{k}+1\right)\right] \\
& \leqq 2^{n}\left(\prod_{\imath=1}^{n-1} \frac{X}{e^{k_{i}}}\right) \frac{X e^{k_{1}+\cdots+k_{n-1}+n-1}}{k} \\
& =(2 X)^{n} e^{n-1} / k .
\end{aligned}
$$

$(* * *)$

Combining $(*),(* *)$ and $(* * *)$, we get

$$
D(S, X)=N(S, X) /(2 X)^{n}=O\left((\log X)^{n-1}\right) .
$$

Thus $S$ is a Danzer set which provides an example for Theorem 2 .

\section{REFERENCES}

1. L. Danzer, Convexity, Proc. Coll., Copenhagen, (1965), 310.

2. J. W. S. Cassels, Introduction to the Geometry of Numbers, Springer-Verlag, 1959.

3. D. B, Sawyer, J. London Math. Soc., 41 (1966), 466-468.

Received July 29, 1970. Research partially supported by NSF Grant GP-9588.

The Ohio State University

AND

Panjab University, Chandigrah, India 



\section{PACIFIC JOURNAL OF MATHEMATICS}

\section{EDITORS}

\author{
H. SAMElson \\ Stanford University \\ Stanford, California 94305 \\ C. R. HOBBY \\ University of Washington \\ Seattle, Washington 98105
}

J. DugundjI

Department of Mathematics University of Southern California Los Angeles, California 90007

RICHARD ARENS

University of California

Los Angeles, California 90024

\section{ASSOCIATE EDITORS}
E. F. BECKENBACH
B. H. NeUmanN
F. WOLE
K. YOSHIDA

\section{SUPPORTING INSTITUTIONS}

\author{
UNIVERSITY OF BRITISH COLUMBIA \\ CALIFORNIA INSTITUTE OF TECHNOLOGY \\ UNIVERSITY OF CALIFORNIA \\ MONTANA STATE UNIVERSITY \\ UNIVERSITY OF NEVADA \\ NEW MEXICO STATE UNIVERSITY \\ OREGON STATE UNIVERSITY \\ UNIVERSITY OF OREGON \\ OSAKA UNIVERSITY \\ UNIVERSITY OF SOUTHERN CALIFORNIA
}

\author{
STANFORD UNIVERSITY \\ UNIVERSITY OF TOKYO \\ UNIVERSITY OF UTAH \\ WASHINGTON STATE UNIVERSITY \\ UNIVERSITY OF WASHINGTON \\ * * * \\ AMERICAN MATHEMATICAL SOCIETY \\ CHEVRON RESEARCH CORPORATION \\ NAVAL WEAPONS CENTER
}

The Supporting Institutions listed above contribute to the cost of publication of this Journal, but they are not owners or publishers and have no responsibility for its content or policies.

Mathematical papers intended for publication in the Pacific Journal of Mathematics should be in typed form or offset-reproduced, (not dittoed), double spaced with large margins. Underline Greek letters in red, German in green, and script in blue. The first paragraph or two must be capable of being used separately as a synopsis of the entire paper. The editorial "we" must not be used in the synopsis, and items of the bibliography should not be cited there unless absolutely necessary, in which case they must be identified by author and Journal, rather than by item number. Manuscripts, in duplicate if possible, may be sent to any one of the four editors. Please classify according to the scheme of Math. Rev. Index to Vol. 39. All other communications to the editors should be addressed to the managing editor, Richard Arens, University of California, Los Angeles, California, 90024.

50 reprints are provided free for each article; additional copies may be obtained at cost in multiples of 50 .

The Pacific Journal of Mathematics is published monthly. Effective with Volume 16 the price per volume (3 numbers) is $\$ 8.00$; single issues, $\$ 3.00$. Special price for current issues to individual faculty members of supporting institutions and to individual members of the American Mathematical Society: $\$ 4.00$ per volume; single issues $\$ 1.50$. Back numbers are available.

Subscriptions, orders for back numbers, and changes of address should be sent to Pacific Journal of Mathematics, 103 Highland Boulevard, Berkeley, California, 94708.

PUBLISHED BY PACIFIC JOURNAL OF MATHEMATICS, A NON-PROFIT CORPORATION

Printed at Kokusai Bunken Insatsusha (International Academic Printing Co., Ltd.), 7-17, Fujimi 2-chome, Chiyoda-ku, Tokyo, Japan. 


\section{Pacific Journal of Mathematics}

\section{Vol. 37, No. $2 \quad$ February, 1971}

Charles Compton Alexander, Semi-developable spaces and quotient images of metric spaces .................................... 277

Ram Prakash Bambah and Alan C. Woods, On a problem of Danzer. . . . . . . . . 295

John A. Beekman and Ralph A. Kallman, Gaussian Markov expectations and related integral equations . ....................................

Frank Michael Cholewinski and Deborah Tepper Haimo, Inversion of the Hankel

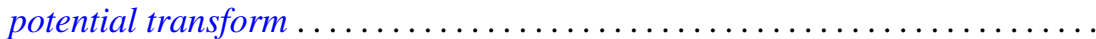

John H. E. Cohn, The diophantine equation

$$
Y(Y+1)(Y+2)(Y+3)=2 X(X+1)(X+2)(X+3) \ldots \ldots \ldots \ldots \ldots
$$

Philip C. Curtis, Jr. and Henrik Stetkaer, A factorization theorem for analytic

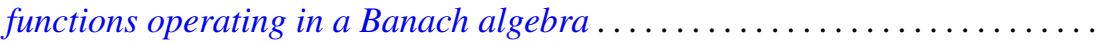

Doyle Otis Cutler and Paul F. Dubois, Generalized final rank for arbitrary limit ordinals

Keith A. Ekblaw, The functions of bounded index as a subspace of a space of entire functions

Dennis Michael Girard, The asymptotic behavior of norms of powers of

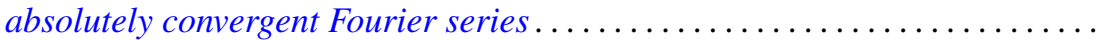

John Gregory, An approximation theory for elliptic quadratic forms on Hilbert spaces: Application to the eigenvalue problem for compact quadratic forms. 383

Paul C. Kainen, Universal coefficient theorems for generalized homology and stable cohomotopy.

Aldo Joram Lazar and James Ronald Retherford, Nuclear spaces, Schauder bases, and Choquet simplexes.

David Lowell Lovelady, Algebraic structure for a set of nonlinear integral operations

John McDonald, Compact convex sets with the equal support property . 429

Forrest Miller, Quasivector topologies

Marion Edward Moore and Arthur Steger, Some results on completability in commutative rings.

A. P. Morse, Taylor's theorem

Richard E. Phillips, Derek J. S. Robinson and James Edward Roseblade, Maximal subgroups and chief factors of certain generalized soluble groups.

Doron Ravdin, On extensions of homeomorphisms to homeomorphisms ...

John William Rosenthal, Relations not determining the structure of $\mathrm{L}$

Prem Lal Sharma, Proximity bases and subbases ........... .

Larry Smith, On ideals in $\Omega_{*}^{u}$. .

Warren R. Wogen, von Neumann algebras generated by operators similar to normal operators

R. Grant Woods, Co-absolutes of remainders of Stone-Čech 